\title{
The Effects of Mindfulness on Older Adults with Mild Cognitive Impairment
}

\author{
Wee Ping Wong ${ }^{\mathrm{a}, *}$, Jan Coles ${ }^{\mathrm{a}}$, Richard Chambers ${ }^{\mathrm{b}}$, David Bin-Chia $\mathrm{Wu}^{\mathrm{c}}$ and Craig Hassed ${ }^{\mathrm{a}}$ \\ ${ }^{a}$ Department of General Practice, School of Primary and Allied Health Care, Faculty of Medicine, \\ Nursing and Health Sciences, Monash University, VIC, Australia \\ ${ }^{\mathrm{b}}$ Counselling and Mental Health Programs, Campus Community Division, Monash University, VIC, Australia \\ ${ }^{\mathrm{c} S}$ Shool of Pharmacy, Monash University Malaysia, Subang Jaya, Selangor, Malaysia
}

Accepted 24 October 2017

\begin{abstract}
.
Background: The current lack of an effective cure for dementia would exacerbate its prevalence and incidence globally. Growing evidence has linked mindfulness to cognitive and psychological improvements that could be relevant for mild cognitive impairment (MCI).

Objective: To investigate whether mindfulness practice can improve health outcomes of MCI.

Methods: The study is the first longitudinal mixed-methods observational study with a one-year follow-up period, that customized an eight-week group-based mindfulness training program for older adults with MCI $(n=14)$. Measures included cognitive function, psychological health, trait mindfulness, adherence to mindfulness practice, and everyday activities functioning as assessed at pre-intervention, post-intervention, and one-year follow-up. Repeated measures ANOVAs, Pearson's correlation analyses, and Mann-Whitney U tests were performed.

Results: The MCI participants showed significant improvements in cognitive function $(p<0.05)$ and trait mindfulness $(p<0.05)$ after completing the intervention. Between program intervention and one-year follow-up (59 weeks), positive correlations were found between their cognitive function $(p<0.05)$ and everyday activities functioning $(p<0.05)$ with the duration of mindfulness meditation; and between trait mindfulness and the level of informal mindfulness practice $(p<0.05)$. Those who meditated more during these 59 weeks, showed greater improvements in cognitive function $(p<0.05)$ and everyday activities functioning $(p<0.05)$, with large effect sizes at the one-year follow-up. Qualitative findings will be reported separately.

Conclusion: Long-term mindfulness practice may be associated with cognitive and functional improvements for older adults with MCI. Mindfulness training could be a potential efficacious non-pharmacological therapeutic intervention for MCI.
\end{abstract}

Keywords: Activities of daily living, Alzheimer's disease, anxiety, cognition, dementia, depression, meditation, mild cognitive impairment, mindfulness

\section{INTRODUCTION}

Against the global backdrop of population aging and medical care advances, the risk of developing neurodegenerative diseases such as dementia

${ }^{*}$ Correspondence to: Wee Ping Wong, Department of General Practice, School of Primary and Allied Health Care, Faculty of Medicine, Nursing and Health Sciences, Monash University, Building 1, 270 Ferntree Gully Road, Notting Hill, VIC 3168, Australia. Tel.: +61 39902 4461; E-mail: ping.wong@monash. edu. increases as people live longer. The World Health Organization (WHO) recently reported that globally, approximately 47 million people have dementia, with an annual incidence of 10 million new cases and a projected increase to almost 75 million people in 2030 and 132 million people by 2050 [1]. The estimated total global cost associated with dementia in 2015 was US\$818 billion, which was equivalent to $1.1 \%$ of the worldwide gross domestic product (GDP) [1]. Consequently, the burden of dementia costs and care cast a ripple effect across the spectrum 
of the patients, families, caregivers, healthcare resources, health systems, society and economy. This public health priority is exacerbated by no current effective cure for dementia or treatment that would alter its progressive trajectory.

Alzheimer's disease (AD) may constitute about $60 \%$ to $70 \%$ of dementia cases as the most common form/cause of dementia [1], while mild cognitive impairment (MCI) can be considered as a prodromal stage of AD. The adjusted annual conversion rate from Mayo defined MCI to $\mathrm{AD}$ was $8.1 \%$ in specialist clinical settings, and $6.8 \%$ in community studies [2]. The symptomatic sub-clinical MCI phase can be considered a timely window where an early non-pharmacological therapeutic intervention such as mindfulness could be implemented.

Mindfulness involves developing awareness through paying attention to the unfolding of ongoing experience in the present moment, purposefully and non-judgmentally [3]. Common types of mindfulness-based interventions (MBIs) encompass the mindfulness-based stress reduction (MBSR) program, mindfulness-based cognitive therapy (MBCT) specifically adapted for depression as well as forms of meditation like Zen and Vipassana (insight) meditations. Mindfulness practices may be categorized in terms of formal practice which involves mindfulness meditation that could incorporate techniques such as 'body scan' and 'breath', and informal practice which basically extends the awareness cultivated during formal practice into everyday activities and daily experiences such as eating, walking and communicating mindfully. In short, mindfulness is an attentional stance and a way of living, while meditation is a method of training mindfulness.

In recent years, a small but growing evidence base has shown the positive effects of mindfulness/meditation on healthy, aged and clinical populations including people with neurodegenerative conditions. Short-term mindfulness training may potentially produce positive effects for meditationnaive participants: cognition, mindfulness and anxiety of randomly assigned young participants improved after four days of mindfulness training [4]; attention, anxiety and depression improved after five days of meditation/mindfulness training [5]; and cognitive function, mindfulness and depressive symptoms improved after a 10-day intensive mindfulness retreat [6]. However, the findings may not be generalizable to the wider population due to the relatively small sample sizes in these studies. Interestingly, older long-term mindfulness practitioners were found to perform better than the young control group in attentional processing, suggesting that agerelated attentional deficits in trained older adults may be offset [7]. Likewise, mindfulness may contribute to a slower decline in fluid intelligence and brain functional architecture, as observed in aged yoga and mindfulness practitioners [8]. The exploratory evidence of the potential beneficial effects of meditation in counteracting age-related cognitive decline suggests the promising feasibility of meditation interventions for older adults [9]. Like memory enhancement training (MET), yoga increased MCI participants' neural connectivity in relation to memory performance, but it also yielded a broader benefit of improved mood [10].

Notably, one of the earliest reliable indicators of $\mathrm{MCI}$ and $\mathrm{AD}$ and a long-term predictor of conversion from MCI to $\mathrm{AD}$, was evident in the hippocampal volume loss or atrophy implicated in memory and cognitive decline [11-13]. By contrast, larger brain and hippocampal volumes could have a neuroprotective effect in preserving the cognitive function of elderly individuals, despite having substantial AD pathology [14]. Several magnetic resonance imaging (MRI) studies demonstrated the pre-post effects of the eight-week MBSR intervention on increasing the gray matter concentration within the left hippocampus of meditation-naive participants [15], and the functional connectivity between the posterior cingulate cortex and bilateral medial prefrontal cortex and left hippocampus of MCI participants [16]. These preliminary neuroimaging findings elucidate the possible impact of mindfulness practice in modulating neurological structure of the hippocampus that would result in better cognitive function and mental capacity. Additionally, cross-sectional MRI studies found hippocampal advantages in experienced mindfulness meditators, compared with controls, such as higher gray matter concentration [17], larger hippocampal volume [18, 19], and reduced age-related atrophy of the left hippocampal subiculum [20, 21]. However, the causal inference of mindfulness leading to these hippocampal benefits may not be made since these meditators' long-term practice might be attributed to other factors such as personal traits, lifestyle and socioeconomic status.

High chronic psychological distress has been shown to be associated with a $40 \%$ greater risk of developing MCI, and to be linked to poorer performance in multiple cognitive functions, particularly episodic memory, and faster cognitive decline [22]. Moreover, high proneness to psychological 
distress doubled the risk of developing $\mathrm{AD}$ due to a ten-fold increase in episodic memory decline [23]. This may be attributed to the burden of prolonged stress and chronic activation of the sympathetic nervous system, adding an allostatic load with the resulting high cortisol secretion and proinflammatory effect leading to atrophy of brain nerve cells, particularly in the hippocampus [24]. However, enlargement of amygdala and atrophy of prefrontal cortex as implicated in psychiatric illnesses such as major depression and mood/anxiety disorders, could be ameliorated with timely therapeutic interventions [24]. Following the eight-week MBSR program, positive correlations were found between participants' perceived stress reductions and decrease of gray matter density in the right amygdala [25], and between meditation-naïve participants' psychological wellbeing and brainstem gray matter concentration [26]. These findings suggest the potential neuroprotective benefits of mindfulness that are corresponded by altered brain morphology and emotion regulation. Notably in a study involving participants with $\mathrm{AD}, \mathrm{MCI}$ and other diagnoses of cognitive impairment, their depression and quality of life improved after completing an eight-week mindfulness program [27]. Mindfulness was shown to reduce the default mental activity of mind-wandering, worry and rumination [28-30] that has been associated with $\mathrm{AD}$ [31] and amyloid- $\beta(A \beta)$ deposition [32], by increasing self-monitoring and cognitive control [28]. However, these studies lack an active control group and a longer follow-up to measure the long-term effects of mindfulness practice on psychological health. On the contrary, stronger evidence from randomized controlled trials (RCTs) found that compared with the music listening control group, participants with subjective cognitive decline (SCD) who practiced Kirtan Kriya (KK) meditation, showed greater improvements in perceived stress and mood at 12 and 26 weeks [33]; and that relative to the MET control group, MCI participants who practiced Kundalini yoga showed better depressive symptoms and resilience at 12 weeks [34].

The MBIs used in recent studies involving participants with MCI and dementia were not customized to their specific needs and health outcomes [35-37]. Despite using the quality study design of a RCT $[35,37]$, the sample sizes in these studies were small without longer follow-up period. Moreover, meaningful interpretation may be restricted by small sample sizes of participants with heterogenous diagnoses of cognitive impairment [27] or at different stages of dementia [36]. Next, emphasizing informal mindfulness practice, that is being attentive in doing the activity at hand and open to the ongoing experience in the present moment may potentially enhance MCI individuals' awareness and cognitive stimulation outside of the formal practice of meditation sessions, and improve their activities of daily living (ADL) functioning. However, past studies have not adequately examined the relationship between informal mindfulness practice and MCI participants' ADL functioning which becomes progressively impaired when MCI is converted to AD. The present study is the first longitudinal mixed-methods observational study that investigates the long-term effects of MCI participants' mindfulness practices after attending a customized eight-week mindfulness training program, incorporating both formal and informal mindfulness practices, with support from a familiar support person (FSP) and the research team, on various health outcomes after the program and in a one-year follow-up.

The aims of the study were to investigate: (1) whether mindfulness practices can improve health outcomes of older adults with MCI, specifically cognitive function, psychological health (depression, anxiety and stress), mindfulness and ADL functioning; (2) whether the degree of any improvement in these health outcomes varies with the duration/ level of formal and informal mindfulness practices; (3) how much of any observed improvement is due to formal practice and how much is due to informal practice; and (4) whether these improvements were maintained over a one-year follow-up period [38].

\section{METHODS}

\section{Study design}

This observational study was an exploratory, mixed-methods, non-randomized longitudinal clinical research project incorporating pre-/postintervention design. A detailed description of the study protocol has been previously published [38]. The current paper presents the study's quantitative data and findings. Qualitative findings will be reported separately. Persons with a clinical diagnosis of MCI given by treating health professionals received an eight-week Mindfulness for MCI program intervention that was developed at Monash University as a part of this study. A follow-up assessment was conducted one year after the program 
to measure the effects of long-term mindfulness practices on older adults with MCI.

\section{Participants}

The MCI participants fulfilled the following study eligibility criteria. The inclusion criteria were:

- Clinical diagnosis of MCI;

- Must be able to give informed consent to participate by signing the Consent Form; and

- At least 60 years old.

The exclusion criteria were:

- Current active or past significant experience with meditation or yoga;

- Current use of prescribed cognitive intervention or electromagnetic stimulation;

- Acquired/traumatic brain injury;

- Started new neurological/psychoactive medication within 3 months prior to the first data collection session;

- Current intake of drugs that significantly alter cognition;

- Illicit drug or alcohol abuse or dependence within the previous 5 years;

- Current intake of cholinesterase inhibitors;

- Current severe psychiatric condition; or neurological/cerebrovascular condition; or chronic medical condition that requires intensive medical treatment and monitoring; or advanced cognitive decline;

- Major impairments in eyesight, hearing or upper limb motor movements; or

- English language difficulties.

\section{Recruitment}

The MCI participants were recruited through the Cognitive, Dementia and Memory Services (CDAMS), specialist and General Practice (GP) clinics, Alzheimer's Australia Victoria (recently renamed as Dementia Australia), and the general community that responded to advertisements of the study in local newspapers, social media and Monash University. All MCI participants provided voluntary written informed consent to participate, in the presence of a FSP who must be a family member above the age of 18 years old, with no English language difficulties. The FSP confirmed the MCI participant's capacity for informed consent, supported his/her participation, and provided information about his/her functional abilities, and demographic, health and lifestyle details.

\section{Intervention}

The intervention was a mindfulness training program that involved the MCI participants (where possible, accompanied by a FSP) attending a 1.5hour group session, once weekly for eight weeks. In addition to the standard care that the MCI participants received from their own treating health professionals, all except one satisfactorily completed the intervention (attended at least six out of the eight weekly sessions). The program was conducted at two different inner suburbs of Melbourne, Australia which were chosen for their proximity to the two CDAMS that referred their clients for this study. All MCI participants and FSPs were each given a mindfulness training program manual, a compact disc containing audio recordings of guided mindfulness practices to support them with their home mindfulness meditation practice, and an AU\$50 gift card to each dyad to compensate for their travel costs. The FSPs were encouraged to use the resources and practice mindfulness with their MCI participant as a show of support.

The intervention was customized to cater to the needs, health outcomes and level of understanding of older adults with MCI and guided by experienced mindfulness facilitators Craig Hassed $(\mathrm{CH})$ and Richard Chambers (RC). The program attendees were taught both formal and informal mindfulness practices. The formal practice involves using specific techniques such as 'body scan' and 'breath' meditations to observe and maintain attention on the object of meditation without judgment and reactivity amidst any distractions of wandering thoughts or uncomfortable emotions, and 'loving-kindness' meditation to cultivate goodwill and kindness towards all beings, including oneself [39]. The informal practice involves extending such attentiveness and awareness to everyday activities such as mindful eating and walking, and doing mental exercises such as reading and solving puzzles. The program content also included the applications of mindfulness relevant to older adults with MCI such as attention and memory, ADL functioning, psychological health, emotion management, learning, mental flexibility and problem solving, and sleep. The MCI participants recorded their mindfulness meditation in minutes on their own or with assistance from their FSP, and rated their informal mindfulness level (hardly at all, sometimes, often, 
most of the time), on the practice record forms across the duration of program intervention and one-year follow-up. Researcher Wee Ping Wong (WW) was present at every weekly program session to record participant attendance and collect practice record forms and observational data; and contact the MCI participants and FSPs by email or phone to follow up the participants' mindfulness practice progress.

\section{Data collection}

This mixed-methods study involves the collection and analyses of quantitative data from cognitive assessment and questionnaires/scales and qualitative data collected through semi-structured interviews, participant observation and follow-up phone calls. Data were collected about two weeks from the three time points: pre-intervention (T1), post-intervention (T2) and one-year follow-up (T3).

\section{Quantitative data}

The primary outcome variable was the change in the MCI participants' cognitive function assessed by the Montreal Cognitive Assessment (MoCA). The MoCA measures eight cognitive domains: visuospatial/executive, naming, memory, attention, language, abstraction, delayed recall, and orientation over time [40]. The MoCA was administered by researcher (WW) during the three data collection sessions.

The secondary outcome variables measured at $\mathrm{T} 1$, $\mathrm{T} 2$, and T3 were changes in the MCI participants' psychological health as measured by the 21-item Depression Anxiety Stress Scales (DASS-21) [41], trait mindfulness as measured by the 14-item Freiburg Mindfulness Inventory (FMI) [42], and adherence to formal and informal mindfulness practices as measured by the 12-item Mindfulness Adherence Questionnaire (MAQ) [38] specifically designed for this study. In addition, the 25-item Bayer Activities of Daily Living Scale (B-ADL) [43, 44], and the Demographic, Health and Lifestyle (DHL) questionnaire [38] adapted from several related questionnaires [45-49] for this study, were completed by the FSPs who provided information about their MCI participant at T1, T2, and T3. FSPs who could not attend the second and/or third data collection sessions together with their MCI participant, returned the completed B-ADLs and DHL questionnaires using reply paid return envelopes.

\section{Quantitative data analyses}

To investigate whether mindfulness practices improve the MCI participants' outcome measures at the three time points, one-way repeated measures analyses of variance (ANOVAs) were conducted to measure the effects of mindfulness practices on each of the dependent variables, namely cognitive function, depression, anxiety, stress, trait mindfulness, informal mindfulness adherence and ADL functioning at T1, T2, and T3, with an experiment-wise error rate of 0.05. Post-hoc tests using Bonferroni confidence interval adjustment were conducted to establish which pair of time points was significantly different for each dependent variable for which the ANOVA yielded a significant effect. Given the exploratory nature of the study with a very small sample size, a nominal error rate of 0.05 instead of the Bonferroni-adjusted Type I error rate 0.007 was used to identify any trends to guide further research investigations with a much larger sample. It was also due to these constraints that multivariate analysis of variance was not used.

To examine whether the degree to which any improvement in the outcome measures varies with the duration/level of formal and informal mindfulness practices, Pearson product-moment correlation coefficient analyses were conducted to investigate any relationship between the score improvement in each of these outcome measures with the average weekly length of mindfulness meditation (in minutes), and with the average weekly informal mindfulness practice rating at $\mathrm{T} 1, \mathrm{~T} 2$, and $\mathrm{T} 3$.

Additionally, Mann-Whitney U tests were conducted to examine differences between MCI participants who practiced below and above the average weekly length of mindfulness meditation (formal practice), as well as between those who rated below and above the average weekly informal mindfulness level. Only study data that were collected from MCI participants who satisfactorily completed the intervention, were analyzed for meaningful data interpretation. The time intervals for data analyses between $\mathrm{T} 1$ and $\mathrm{T} 2$, between $\mathrm{T} 2$ and $\mathrm{T} 3$ and between $\mathrm{T} 1$ and $\mathrm{T} 3$ were seven weeks, 52 weeks, and 59 weeks, respectively.

\section{Ethical clearance, trial registration, participant consent}

This study was approved by the Melbourne Health Human Research Ethics Committee (Project number: 
HREC/14/MH/324), and conducted in accord with the National Statement on Ethical Conduct in Human Research (2007). The study was registered with the Australian New Zealand Clinical Trials Registry on 1 August 2014 (Trial ID: ACTRN12614000820606). All MCI participants and FSPs provided written informed consent prior to study involvement.

\section{RESULTS}

A total of 14 MCI participants were initially recruited into the observational study. However, one of them dropped out after attending the first mindfulness training session. Thirteen MCI participants satisfactorily completed the intervention and contributed to T1 and T2 study data. The demographic characteristics of the 13 MCI participants are presented in Table 1. As one MCI participant later withdrew her participation a few months after completing the intervention, $\mathrm{T} 3$ study data were collected from the remaining 12 MCI participants only. In short, data at all three time points were available for only 12 MCI participants. The MAQ - Formal data relating to the MCI participants' adherence to formal mindfulness practice were unavailable for preintervention analyses at $\mathrm{T} 1$ as they only learnt and practiced mindfulness meditation after they started the program intervention. There were no adverse events reported in relation to the study protocol.

Table 1

Demographic characteristics of MCI participants

\begin{tabular}{lr}
\hline Demographic characteristics of MCI participants $(n=13)$ \\
\hline Gender & \\
Female, \% (n) & $61.5 \%(8)$ \\
$\quad$ Male, \% (n) & $38.5 \%(5)$ \\
Age, y & $76.5(6.7)$ \\
$\quad$ Mean (SD) & \\
Age range & $23.1 \%(3)$ \\
60-69, \% (n) & $38.5 \%(5)$ \\
$70-79, \%(\mathrm{n})$ & $38.5 \%(5)$ \\
80-89, \% (n) & \\
Highest education level & $30.8 \%(4)$ \\
Secondary school, \% (n) & $15.4 \%(2)$ \\
Diploma, \% (n) & $23.1 \%(3)$ \\
Bachelor degree, \% (n) & $30.8 \%(4)$ \\
Postgraduate, \% (n) & $0 \%(0)$ \\
Current smoker & \\
$\quad \%(\mathrm{n})$ & $61.5 \%(8)$ \\
Marital status & $15.4 \%(2)$ \\
Married, \% (n) & $23.1 \%(3)$ \\
Divorced, \% (n) & \\
Widowed, \% (n) & \\
The table shows the demographic characteristics of the 13 \\
MCI participants who satisfactorily completed the inter- \\
vention.
\end{tabular}

Effects of mindfulness practices on outcome measures over time, repeated measures ANOVAs

One-way repeated measures ANOVAs were conducted to compare scores on the MoCA, DASS-21, FMI, MAQ - Informal, and B-ADL over time at $\mathrm{T} 1, \mathrm{~T} 2$, and $\mathrm{T} 3$. The descriptive statistics are presented in Table 2 . The effects of doing mindfulness practices for 7 weeks during the intervention period, on the MCI participants' cognitive function and trait mindfulness were statistically significant. Cognitive function as measured by MoCA significantly improved over time $(p=0.03)$, and showed a very large effect size (multivariate partial eta squared $=0.52$ ) [50]. Based on pairwise comparisons, there was a mean increase of 1.83 MoCA points $(p=0.04)$ in cognitive function after attending the mindfulness training program and doing mindfulness practices for 7 weeks. Trait mindfulness as measured by FMI significantly increased over time $(p=0.01)$, and showed a very large effect size (multivariate partial eta squared $=0.58$ ) [50]. Pairwise comparisons showed a mean increase of $3.75 \mathrm{FMI}$ points $(p=0.02)$ in trait mindfulness after doing mindfulness practices for the same duration of 7 weeks. Table 3 shows the repeated measures ANOVA on the MCI participants' outcome measures at all three time points.

\section{Relationships between improvements in outcome measures and duration/level of mindfulness practices, Pearson's correlations}

The linear associations between the amount of improvements in each of the scores of MoCA, DASS21, FMI, MAQ - Informal, and B-ADL at T1, T2, and $\mathrm{T} 3$ and average weekly length of mindfulness meditation (in minutes), and average weekly informal mindfulness practice rating (hardly at all, sometimes, often, most of the time) were investigated using Pearson product-moment correlation coefficient analyses. A significant negative correlation between post-intervention improvement in cognitive function and average weekly informal mindfulness practice rating over 7 weeks between $\mathrm{T} 1$ and $\mathrm{T} 2$ was observed at T2 $(p=0.02)$ (Table 4).

Additionally, the only significant positive correlations observed at T3 after the MCI participants attended the program and practiced mindfulness for 59 weeks were between (1) overall improvement in cognitive function and average weekly length of mindfulness meditation ( $p=0.045)$; (2) overall improvement in trait mindfulness and average weekly 
Table 2

Descriptive statistics for scores on the MoCA, DASS-21, FMI, MAQ - Informal and B-ADL for T1, T2, and T3

\begin{tabular}{|c|c|c|c|c|c|c|c|c|c|}
\hline \multirow[b]{2}{*}{ Outcome measure } & \multicolumn{3}{|c|}{ T1 } & \multicolumn{3}{|c|}{$\mathrm{T} 2$} & \multicolumn{3}{|c|}{ T3 } \\
\hline & $\mathrm{n}$ & Mean & $\begin{array}{l}\text { Standard } \\
\text { deviation }\end{array}$ & $\mathrm{n}$ & Mean & $\begin{array}{l}\text { Standard } \\
\text { deviation }\end{array}$ & $\mathrm{n}$ & Mean & $\begin{array}{l}\text { Standard } \\
\text { deviation }\end{array}$ \\
\hline $\mathrm{MoCA}$ & 12 & 24.00 & 3.16 & 12 & 25.83 & 3.04 & 12 & 24.75 & 3.75 \\
\hline DASS - D & 12 & 4.42 & 3.06 & 12 & 3.25 & 2.60 & 12 & 3.33 & 2.71 \\
\hline DASS - A & 12 & 2.92 & 2.23 & 12 & 2.42 & 1.78 & 12 & 2.75 & 2.14 \\
\hline DASS $-S$ & 12 & 5.42 & 2.75 & 12 & 5.00 & 1.76 & 12 & 5.17 & 3.10 \\
\hline FMI & 12 & 39.25 & 5.69 & 12 & 43.00 & 4.84 & 12 & 42.33 & 7.11 \\
\hline MAQ - Informal & 12 & 19.00 & 6.19 & 12 & 21.42 & 3.97 & 12 & 20.00 & 4.63 \\
\hline B-ADL & 12 & 2.57 & 0.74 & 12 & 2.29 & 0.70 & 12 & 3.00 & 1.10 \\
\hline
\end{tabular}

The table shows the descriptive statistics for scores on the respective outcome measures of the remaining 12 MCI participants for all three time points. MoCA, Montreal Cognitive Assessment; DASS-21, 21-item Depression Anxiety Stress Scales; FMI, Freiburg Mindfulness Inventory; MAQ, Mindfulness Adherence Questionnaire; B-ADL, Bayer Activities of Daily Living Scale; T1, Pre-intervention; T2, Post-intervention; T3, One-year follow-up; DASS - D, DASS - Depression; DASS - A, DASS - Anxiety; DASS - S, DASS - Stress.

Table 3

Repeated measures ANOVAs on 12 MCI participants' outcome measures at three time points

\begin{tabular}{|c|c|c|c|c|c|c|c|}
\hline Outcome measure & F & Hypothesis df & Error $\mathrm{df}$ & $p$-value & $\begin{array}{l}\text { Mauchly's test } \\
\text { of sphericity } \\
\text { Sig. (Greenhouse- } \\
\text { Geisser Sig.) }\end{array}$ & $\begin{array}{c}\text { Pairwise } \\
\text { comparisons } \\
\text { Mean difference } \\
\text { between } \mathrm{T} 2 \text { and } \mathrm{T} 1 \\
(p \text {-value })\end{array}$ & $\begin{array}{c}\text { Pairwise } \\
\text { comparisons } \\
\text { Mean difference } \\
\text { between T3 and T1 } \\
(p \text {-value })\end{array}$ \\
\hline $\mathrm{MoCA}$ & 5.38 & 2 & 10 & $0.03 *$ & 0.37 & $1.83(0.04 *)$ & $0.75(0.80)$ \\
\hline DASS - Depression & 1.33 & 2 & 10 & 0.31 & 0.74 & $-1.17(0.61)$ & $-1.08(0.54)$ \\
\hline DASS - Anxiety & 0.26 & 2 & 10 & 0.78 & 0.56 & $-0.50(1.00)$ & $-0.17(1.00)$ \\
\hline DASS - Stress & 0.37 & 2 & 10 & 0.70 & 0.051 & $-0.42(1.00)$ & $-0.25(1.00)$ \\
\hline FMI & 6.86 & 2 & 10 & 0.01* & 0.44 & $3.75(0.02 *)$ & $3.08(0.10)$ \\
\hline MAQ - Informal & 4.56 & 2 & 10 & 0.04 & $0.01^{\wedge}(0.21)$ & $2.42(0.31)$ & $1.00(1.00)$ \\
\hline B-ADL & 2.31 & 2 & 10 & 0.15 & $0.04^{\wedge}(0.15)$ & $-0.28(0.47)$ & $0.41(0.93)$ \\
\hline
\end{tabular}

The table shows the analyses of variance for repeated measures on the $12 \mathrm{MCI}$ participants in which significant differences were observed in their cognitive function and trait mindfulness between T1 and T2 $(p<0.05)$. $^{\wedge}$ indicates the violation of the assumption of sphericity $(p<0.05)$. As the Greenhouse-Geisser $p$-value of MAQ - Informal is more than 0.05, the difference observed in MAQ - Informal is not considered as significant. ${ }^{*} p<0.05 ; \mathrm{n}=12$; ANOVA, Analysis of variance; df, Degrees of freedom; T1, Pre-intervention; T2, Post-intervention; T3, Oneyear follow-up; MoCA, Montreal Cognitive Assessment; DASS, Depression Anxiety Stress Scales; FMI, Freiburg Mindfulness Inventory; MAQ, Mindfulness Adherence Questionnaire; B-ADL, Bayer Activities of Daily Living Scale.

informal mindfulness practice rating $(p=0.02)$; and (3) overall improvement in ADL functioning and average weekly length of mindfulness meditation $(p=0.04)$, between T1 and T3 (Table 5).

\section{Effects of duration/level of mindfulness practices on improvements in outcome measures, Mann-Whitney U tests}

Mann-Whitney U tests revealed statistically significant differences at various time points in the improvements in cognitive function, formal mindfulness adherence and ADL functioning for the MCI participants who practiced above the average weekly length of mindfulness meditation. First, there was greater cognitive function improvement between $\mathrm{T} 1$ and $\mathrm{T} 3$ for the group that practiced above the average weekly length of mindfulness meditation $(p=0.02)$, with a large effect size $(r=0.69)$ [50], compared with the group that practiced below the average duration. Second, there was also greater improvement in formal mindfulness adherence between $\mathrm{T} 2$ and T3 for the group that practiced above the average weekly length of mindfulness meditation $(p=0.04)$, with a large effect size $(r=0.61)$ [50], compared with the group that practiced below the average duration. Third, there was greater ADL functioning improvement between $\mathrm{T} 1$ and $\mathrm{T} 3$ for the group that practiced above the average weekly length of mindfulness meditation $(p=0.04)$, with a large effect size $(r=0.60)$ [50], compared with the group that practiced below the average duration.

Additionally, Mann-Whitney $U$ test also found greater trait mindfulness improvement between $\mathrm{T} 1$ and T3 for the group that rated above the average weekly informal mindfulness level $(p=0.02)$, with a large effect size $(r=0.68)$ [50], compared with the group that rated below the average level. 
Table 4

Pearson product-moment correlations between improvements in outcome measures and mindfulness practices at $\mathrm{T} 2$

\begin{tabular}{lcc}
\hline Improvements in outcome measures at T2 & $\begin{array}{c}\mathrm{T} 2-\mathrm{T} 1 \\
\text { 7-week average weekly } \\
\text { length of mindfulness } \\
\text { meditation (in minutes })\end{array}$ & $\begin{array}{c}\mathrm{T} 2-\mathrm{T} 1 \\
\text { 7-week average weekly } \\
\text { informal mindfulness } \\
\text { practice rating }\end{array}$ \\
$\begin{array}{c}\text { Pearson correlation } r \\
(p \text {-value })\end{array}$ & $\begin{array}{c}\text { Pearson correlation } r \\
(p \text {-value })\end{array}$ \\
\hline $\begin{array}{l}\text { Post-intervention improvement in cognitive } \\
\text { function (MoCA) }\end{array}$ & $0.12(0.71)$ & $\mathbf{- 0 . 6 5 ( \mathbf { 0 . 0 2 } )}$ \\
$\begin{array}{l}\text { Post-intervention improvement in depression } \\
\text { (DASS - Depression) }\end{array}$ & $-0.01(0.99)$ & $-0.06(0.84)$ \\
$\begin{array}{l}\text { Post-intervention improvement in anxiety } \\
\text { (DASS - Anxiety) }\end{array}$ & $0.29(0.34)$ & $-0.29(0.34)$ \\
$\begin{array}{l}\text { Post-intervention improvement in stress (DASS } \\
- \text { Stress) }\end{array}$ & $0.47(0.10)$ & $-0.12(0.70)$ \\
$\begin{array}{l}\text { Post-intervention improvement in trait } \\
\text { mindfulness (FMI) }\end{array}$ & $0.03(0.92)$ & $0.47(0.10)$ \\
$\begin{array}{l}\text { Post-intervention improvement in informal } \\
\text { mindfulness adherence (MAQ - Informal) }\end{array}$ & $0.01(0.96)$ & $-0.28(0.36)$ \\
$\begin{array}{l}\text { Post-intervention improvement in ADL } \\
\text { functioning (B-ADL) }\end{array}$ & $0.51(0.08)$ & $-0.03(0.94)$ \\
\hline
\end{tabular}

The table shows the correlations between improvements in outcome measures and mindfulness practices at $\mathrm{T} 2$. The only significant negative correlation was between post-intervention improvement in cognitive function and average weekly informal mindfulness practice rating over 7 weeks between T1 and T2. ${ }^{*} p<0.05 ; \mathrm{n}=13$; T2, Postintervention; MoCA, Montreal Cognitive Assessment; DASS, Depression Anxiety Stress Scales; FMI, Freiburg Mindfulness Inventory; MAQ, Mindfulness Adherence Questionnaire; B-ADL, Bayer Activities of Daily Living Scale.

Table 5

Pearson product-moment correlations between improvements in outcome measures and mindfulness practices for 59 weeks

Overall Improvements in outcome measures between T1 and T3

\begin{tabular}{|c|c|}
\hline $\begin{array}{c}\mathrm{T} 3-\mathrm{T} 1 \\
\text { Average weekly length of } \\
\text { mindfulness meditation } \\
\text { (in minutes) }\end{array}$ & $\begin{array}{c}\mathrm{T} 3-\mathrm{T} 1 \\
\text { Average weekly informal } \\
\text { mindfulness practice } \\
\text { rating }\end{array}$ \\
\hline $\begin{array}{c}\text { Pearson correlation } r \\
(p \text {-value })\end{array}$ & $\begin{array}{c}\text { Pearson correlation } r \\
(p \text {-value })\end{array}$ \\
\hline $0.59\left(0.045^{*}\right)$ & $-0.05(0.88)$ \\
\hline$-0.17(0.61)$ & $0.11(0.73)$ \\
\hline $0.19(0.56)$ & $-0.20(0.53)$ \\
\hline$-0.13(0.70)$ & $0.03(0.94)$ \\
\hline $0.23(0.48)$ & $0.65(0.02 *)$ \\
\hline $0.30(0.34)$ & $0.06(0.85)$ \\
\hline $0.59(0.04 *)$ & $-0.06(0.85)$ \\
\hline
\end{tabular}

Overall improvement in cognitive function (MoCA)

Overall improvement in depression (DASS - Depression)

Overall improvement in anxiety (DASS - Anxiety)

Overall improvement in stress (DASS - Stress)

Overall improvement in trait mindfulness (FMI)

Overall improvement in informal mindfulness adherence

(MAQ - Informal)

Overall improvement in ADL functioning (B-ADL)

$0.59(0.04 *)$

$-0.06(0.85)$

The table shows the correlations between overall improvements in outcome measures and mindfulness practices at $\mathrm{T} 3$. The following significant positive correlations were observed at T3 between (1) overall improvement in cognitive function and average weekly length of mindfulness meditation; (2) overall improvement in trait mindfulness and average weekly informal mindfulness practice rating; and (3) overall improvement in ADL functioning and average weekly length of mindfulness meditation, over 59 weeks between T1 and T3. ${ }^{*} p<0.05 ; \mathrm{n}=12$; T1, Pre-intervention; T3, One-year follow-up; MoCA, Montreal Cognitive Assessment; DASS, Depression Anxiety Stress Scales; FMI, Freiburg Mindfulness Inventory; MAQ, Mindfulness Adherence Questionnaire; B-ADL, Bayer Activities of Daily Living Scale.

\section{DISCUSSION}

The present study tested the following hypotheses: (1) Mindfulness practices will improve the health outcomes of MCI participants, specifically cognitive function, depression, anxiety, stress, trait mindfulness and ADL functioning; and (2) The degree of improvements in these health outcomes will correlate positively with the duration/level of the MCI participants' formal and informal mindfulness practices.

The MCI participants showed significant improvements in the measures of cognitive function and trait mindfulness after completing the intervention. 
This positive finding of improved cognitive function was similar to the findings of a lesser deterioration in objective memory of older adults with MCI after attending an eight-week MBI [37], of improved cognition of elderly MCI individuals after a three-month Mindful Awareness Practice (MAP) intervention [51] and of improved memory and executive functioning for MCI participants randomized to a three-month Kundalini yoga intervention [34]. As expected, the present finding of an increase in MCI participants' self-reported mindfulness level after the program intervention is consistent with those from other studies $[4,6]$.

Next, positive changes in MCI participants' cognitive function and $\mathrm{ADL}$ functioning correlated significantly with longer duration of formal practice of mindfulness meditation during the 59 weeks between program intervention and one-year followup, suggesting that the effects of mindfulness acquired from meditations over time could be usefully applied to everyday activities. This may suggest that those who persevered with their formal practice, may have potentially ameliorated the cognitive and functional decline of their neurodegenerative MCI condition at one-year follow-up. These findings are consistent with previous studies that suggest the 'spill over' of benefits gained from formal practice, to situations away from the 'meditation cushion'. Hodgins and Adair found that enhanced visual attentional processing cultivated from regular meditation extended outside the practitioners' formal meditation practice [52]. Notably, enduring increased neural functional connectivity involved in cognitive skills, was even observed in the non-meditative (resting) states of experienced long-term meditators [53].

Additionally, in the present study the MCI participants' trait mindfulness was strongly, positively correlated with their rating of informal mindfulness practice during the 59 weeks between program intervention and one-year follow-up. Moreover, the participants who rated their informal mindfulness practice higher than the average level over these 59 weeks, showed greater improvement in trait mindfulness at the one-year follow-up. These findings seem to suggest a congruence between self-insight of awareness in everyday activities and self-reported mindfulness.

Unexpectedly, a strong, negative correlation was found between the MCI participants' cognitive function and their rating of informal mindfulness practice at post-intervention after attending the mindfulness training program. It is possible that the MCI participants with better cognitive function were initially more stringent and conservative in assessing their own awareness level during everyday activities for the first 7 weeks.

As hypothesized, MCI participants who meditated above the average weekly length of mindfulness meditation during the 59 weeks between program intervention and one-year follow-up, showed greater improvements in cognitive function and ADL functioning at the one-year follow-up, compared with those who meditated below the average duration. Furthermore, those who practiced mindfulness meditation more than the average duration for 52 weeks since completing the intervention, showed more formal mindfulness adherence at the one-year follow-up. While it might be argued that these participants' cognitive improvements could be attributed to repeated testings, this appears unlikely as MoCA has a high test-retest reliability [40]. Besides, since the participants' diagnosed cognitive impairment usually implicates short-term memory loss in MCI, even if they had prepared, practiced and memorized the cognitive tasks in the MoCA prior to T2 and T3 data collection sessions, improvements in their cognitive function through better short-term memory may contribute to higher MoCA scores. Moreover, this finding of cognitive improvements was corroborated by the FSPs' assessment of their MCI participant's functional abilities, and those MCI participants who did longer mindfulness meditations, seemed to reap the benefits of greater cognitive and functional improvements. Our findings appear comparable to other studies involving participants with cognitive impairments, for example, older adults with SCD who practiced KK meditation, maintained their improved memory function and cognitive performance at six months [54]. Likewise, MCI participants who practiced Kundalini yoga sustained their improved executive functioning at six months, compared with the MET control group [34]. Additionally, compared with the cognitive stimulation group and progressive muscle relaxation group, the cognitive function of AD patients treated with donepezil stabilized after maintaining mindfulness practice for two years, while recognizing that the $\mathrm{AD}$ cognitive impairment of this participant group was at a more advanced stage [55]. Taken together, the present study adds to the growing evidence that mindfulness practice is related to cognitive function, rather than suggesting a causal relationship that mindfulness practice leads to cognitive improvement. Thus, further research is needed to 
determine if from the outset, older adults who could do more mindfulness practices, were different from those who did less, while adjusting for variables such as age, gender, education, lifestyle, socio-economic status and social/family support.

Contrary to findings from other studies $[4,6$, $25,33,34]$, the psychological health outcome measures of depression, anxiety and stress did not show any significant improvements in this study. This may be due to some MCI participants facing difficult personal circumstances or distressing situations recent to or on the day of the data collection sessions during which such information was not specifically collected. The MCI participants were not extremely severely depressed, anxious or stressed at T1 data collection session, thus it is useful that the findings did not indicate a deterioration in psychological health as a consequence of mindfulness practices.

The study findings propose possible implications for future Mindfulness for MCI programs to include facilitated refresher sessions to reinforce the MCI older adults' understanding and memory of mindfulness knowledge, with an emphasis on formal practice of mindfulness meditation, as well as to encourage accountability of maintaining long-term mindfulness practices. Where possible, half-yearly health outcomes measurements can be taken to provide feedback to the MCI older adults, and minimize the impact of psychological fluctuations on the outcome measures.

\section{Study strengths}

To our knowledge, the present study is the first longitudinal mixed-methods research project that customized a mindfulness program intervention incorporating both formal and informal practices for older adults with MCI, accompanied by their FSP where possible, with a one-year follow-up. Unlike other studies that recruited a mixture of participants with different diagnoses or later stages of cognitive impairment, this study specifically targeted older adults with MCI as the MCI stage is considered a timely therapeutic window when their cognitive ability may still allow learning and retention of new knowledge/skills, and this sub-clinical patient group may be more motivated to act to improve their own health outcomes. The research team involved the FSPs in data collection to obtain their perspective and assessment about their MCI participant; and worked with the FSPs individually to monitor the MCI participants' progress for more than a year to better evaluate the long-term effects of mindfulness practices. The mixed-methods study design enables analyses of qualitative data collected from the semi-structured interviews, participant observation and follow-up phone calls to supplement the quantitative findings in adding value into enhancing the quality of future Mindfulness for MCI programs.

\section{Study limitations}

The major limitations of the study were the extremely small sample size with no active control group. While this was unavoidable because of the availability of suitable participants, it necessitated the use of data analysis procedures that were not ideal. Multivariate analysis of variance and/or multiple regression procedures would not have yielded stable findings. Instead, multiple ANOVAs were used with a nominal Type I error rate of 0.05 . While this may have increased the likelihood of higher experimentwise error rate, it was felt that, in an exploratory study of this kind, the detection of possible trends was of theoretical and practical importance. But Bonferroni confidence interval adjustment was used to determine any significant comparisons in the post-hoc tests following ANOVA. Given the small sample size and the number of Bonferroni adjusted post-hoc tests conducted, the statistically significant findings from the repeated measures ANOVAs may only serve as an indication of trends that need confirmation in future research. We exercised caution in asserting strong confirmation of our research hypotheses. The small sample size should be recognized also as inadequate for establishing stable correlations from which firm conclusions are drawn.

In sum, statistically significant findings from the study should be treated as indicators of what may be true in the general MCI population. They require follow-up confirmatory research. In this context, however, it may be noted that most of the findings are consistent with those from previous studies. Notwithstanding, the study did not establish the plausible mechanisms of action to explain any changes that were observed.

\section{Conclusions}

Altogether, this observational study yielded further evidence for the potential benefits of long-term mindfulness practice as a feasible and efficacious 
non-pharmacological therapeutic intervention option for older adults with MCI. The key findings of potential improvements in cognitive function and everyday activities functioning associated with long-term mindfulness practices may have clinical implications of MCI older adults' better self-management of this neurodegenerative MCI condition, and practical relevance of reducing the burden of care experienced by their support person and family.

While the findings in this study were preliminary yet promising, they should be supplemented with more data from future large longitudinal RCTs involving a mindfulness training group and an active matched control group such as physical activity/exercise group. Biomarker measures can be incorporated in future study designs to more objectively investigate the neural mechanisms of action and optimal dosing of mindfulness practices; and compare how different interventions influence the trajectory of MCI condition. More conclusive findings could subsequently support the proposal of the customized Mindfulness for MCI program at a population-based level.

\section{ACKNOWLEDGMENTS}

The study was supported by the Department of General Practice, Monash University; the Caulfield Hospital CDAMS of Alfred Health; and the Sunshine Hospital CDAMS of Western Health. The study was funded using the General Operating Fund and Consultancy Fund of the Department of General Practice, Monash University; and the first author (WW) was supported through an Australian Government Research Training Program Scholarship.

We gratefully acknowledge our MCI participants and FSPs for their study involvement, and Emeritus Professor Kim Tee Ng for his statistical advice.

\section{CONFLICT OF INTEREST}

Authors $\mathrm{CH}$ and $\mathrm{RC}$ who are mindfulness experts and Monash University Mindfulness Consultants, were involved in the design of the Mindfulness for MCI program, the creation of the MAQ, and the facilitation of the program intervention. To avoid any conflicts of interest, they were blinded to the identifiable data; and the data analyses and interpretation were done in a fair and appropriate way, and totally independent from them.

\section{REFERENCES}

[1] WHO, Dementia Fact sheet, World Health Organization Media center, http://www.who.int/mediacentre/factsheets/ fs362/en/, September 2017, Accessed 16 October 2017.

[2] Mitchell AJ, Shiri-Feshki M (2009) Rate of progression of mild cognitive impairment to dementia-meta-analysis of 41 robust inception cohort studies. Acta Psychiatr Scand 119, 252-265.

[3] Kabat-Zinn J (2003) Mindfulness-based interventions in context: Past, present, and future. Clin Psychol Sci Pract 10, 144-156.

[4] Zeidan F, Johnson SK, Diamond BJ, David Z, Goolkasian $P$ (2010) Mindfulness meditation improves cognition: Evidence of brief mental training. Conscious Cogn 19, 597-605.

[5] Tang YY, Ma Y, Wang J, Fan Y, Feng S, Lu Q, Yu Q, Sui D, Rothbart MK, Fan M, Posner MI (2007) Short-term meditation training improves attention and self-regulation. Proc Natl Acad Sci U S A 104, 17152-17156.

[6] Chambers R, Lo BCY, Allen NB (2008) The impact of intensive mindfulness training on attentional control, cognitive style, and affect. Cogn Ther Res 32, 303-322.

[7] van Leeuwen S, Müller NG, Melloni L (2009) Age effects on attentional blink performance in meditation. Conscious Cogn 18, 593-599.

[8] Gard T, Taquet M, Dixit R, Hölzel BK, de Montjoye YA, Brach N, Salat DH, Dickerson BC, Gray JR, Lazar SW (2014) Fluid intelligence and brain functional organization in aging yoga and meditation practitioners. Front Aging Neurosci 6, 76.

[9] Gard T, Hölzel BK, Lazar SW (2014) The potential effects of meditation on age-related cognitive decline: A systematic review. Ann N Y Acad Sci 1307, 89-103.

[10] Eyre HA, Acevedo B, Yang H, Siddarth P, Van Dyk K, Ercoli L, Leaver AM, Cyr NS, Narr K, Baune BT, Khalsa DS, Lavretsky H (2016) Changes in neural connectivity and memory following a yoga intervention for older adults: A pilot study. J Alzheimers Dis 52, 673-684.

[11] Jack CR, Petersen RC, Xu YC, O'Brien PC, Smith GE, Ivnik RJ, Boeve BF, Waring SC, Tangalos EG, Kokmen E (1999) Prediction of AD with MRI-based hippocampal volume in mild cognitive impairment. Neurology 52, 1397-1397.

[12] Fotuhi M, Do D, Jack C (2012) Modifiable factors that alter the size of the hippocampus with ageing. Nat Rev Neurol 8 , 189-202.

[13] Wang Y, Risacher SL, West JD, McDonald BC, Magee TR, Farlow MR, Gao S, O'Neill DP, Saykin AJ (2013) Altered default mode network connectivity in older adults with cognitive complaints and amnestic mild cognitive impairment. $J$ Alzheimers Dis 35, 751-760.

[14] Erten-Lyons D, Woltjer RL, Dodge H, Nixon R, Vorobik R, Calvert JF, Leahy M, Montine T, Kaye J (2009) Factors associated with resistance to dementia despite high Alzheimer disease pathology. Neurology 72, 354-360.

[15] Hölzel BK, Carmody J, Vangel M, Congleton C, Yerramsetti SM, Gard T, Lazar SW (2011) Mindfulness practice leads to increases in regional brain gray matter density. Psychiatry Res 191, 36-43.

[16] Wells RE, Yeh GY, Kerr CE, Wolkin J, Davis RB, Tan Y, Spaeth R, Wall RB, Walsh J, Kaptchuk TJ, Press D, Phillips RS, Kong J (2013) Meditation's impact on default mode network and hippocampus in mild cognitive impairment: A pilot study. Neurosci Lett 556, 15-19.

[17] Holzel BK, Ott U, Gard T, Hempel H, Weygandt M, Morgen K, Vaitl D (2008) Investigation of mindfulness 
meditation practitioners with voxel-based morphometry. Soc Cogn Affect Neurosci 3, 55-61.

[18] Luders E, Toga AW, Lepore N, Gaser C (2009) The underlying anatomical correlates of long-term meditation: Larger hippocampal and frontal volumes of gray matter. Neuroimage 45, 672-678.

[19] Luders E, Thompson PM, Kurth F, Hong JY, Phillips OR, Wang Y, Gutman BA, Chou YY, Narr KL, Toga AW (2013) Global and regional alterations of hippocampal anatomy in long-term meditation practitioners. Hum Brain Mapp 34, 3369-3375.

[20] Luders E, Kurth F, Toga AW, Narr KL, Gaser C (2013) Meditation effects within the hippocampal complex revealed by voxel-based morphometry and cytoarchitectonic probabilistic mapping. Front Psychol 4, 398.

[21] Kurth F, Cherbuin N, Luders E (2015) Reduced age-related degeneration of the hippocampal subiculum in long-term meditators. Psychiatry Res 232, 214-218.

[22] Wilson RS, Schneider JA, Boyle PA, Arnold SE, Tang Y, Bennett DA (2007) Chronic distress and incidence of mild cognitive impairment. Neurology 68, 2085-2092.

[23] Wilson RS, Evans DA, Bienias JL, Mendes De Leon CF, Schneider JA, Bennett DA (2003) Proneness to psychological distress is associated with risk of Alzheimer's disease. Neurology 61, 1479-1485.

[24] McEwen BS (2004) Protection and damage from acute and chronic stress: Allostasis and allostatic overload and relevance to the pathophysiology of psychiatric disorders. Ann N Y Acad Sci 1032, 1-7.

[25] Hölzel BK, Carmody J, Evans KC, Hoge EA, Dusek JA, Morgan L, Pitman RK, Lazar SW (2010) Stress reduction correlates with structural changes in the amygdala. Soc Cogn Affect Neurosci 5, 11-17.

[26] Singleton O, Hölzel BK, Vangel M, Brach N, Carmody J, Lazar SW (2014) Change in brainstem gray matter concentration following a mindfulness-based intervention is correlated with improvement in psychological well-being. Front Hum Neurosci 8, 33.

[27] Paller KA, Creery JD, Florczak SM, Weintraub S, Mesulam MM, Reber PJ, Kiragu J, Rooks J, Safron A, Morhardt D, O'Hara M, Gigler KL, Molony JM, Maslar M (2015) Benefits of mindfulness training for patients with progressive cognitive decline and their caregivers. Am J Alzheimers Dis 30, 257-267.

[28] Brewer JA, Worhunsky PD, Gray JR, Tang YY, Weber J, Kober H (2011) Meditation experience is associated with differences in default mode network activity and connectivity. Proc Natl Acad Sci U S A 108, 20254-20259.

[29] Zhao X-H, Wang P-J, Li C-B, Hu Z-H, Xi Q, Wu W-Y, Tang X-W (2007) Altered default mode network activity in patient with anxiety disorders: An fMRI study. Eur J Radiol 63, 373-378.

[30] Greicius MD, Flores BH, Menon V, Glover GH, Solvason HB, Kenna H, Reiss AL, Schatzberg AF (2007) Resting-state functional connectivity in major depression: Abnormally increased contributions from subgenual cingulate cortex and thalamus. Biol Psychiat 62, 429-437.

[31] Firbank MJ, Blamire AM, Krishnan MS, Teodorczuk A, English P, Gholkar A, Harrison RM, O'Brien JT (2007) Diffusion tensor imaging in dementia with Lewy bodies and Alzheimer's disease. Psychiatry Res 155, 135-145.

[32] Simic G, Babic M, Borovecki F, Hof PR (2014) Early failure of the default-mode network and the pathogenesis of Alzheimer's disease. CNS Neurosci Ther 20, 692-698.
[33] Innes KE, Selfe TK, Khalsa DS, Kandati S (2016) Effects of meditation versus music listening on perceived stress, mood, sleep, and quality of life in adults with early memory loss: A pilot randomized controlled trial. J Alzheimers Dis 52, 1277-1298.

[34] Eyre HA, Siddarth P, Acevedo B, Van Dyk K, Paholpak P, Ercoli L, St Cyr N, Yang H, Khalsa DS, Lavretsky H (2017) A randomized controlled trial of Kundalini yoga in mild cognitive impairment. Int Psychogeriatr 29, 557-567.

[35] Wells RE, Kerr CE, Wolkin J, Dossett M, Davis RB, Walsh J, Wall RB, Kong J, Kaptchuk T, Press D (2013) Meditation for adults with mild cognitive impairment: A pilot randomized trial. J Am Geriatr Soc 61, 642-645.

[36] Leader G, Litherland R, Mason T, Pilchick T, Sansom S, Robertson G (2013) Mindfulness and dementia: Report of a pilot study. Innovations in Dementia and Positive Ageing Associates.

[37] Larouche E, Chouinard A-M, Goulet S, Hudon C (2016) Mindfulness-based intervention prevents memory decline and improves mood and quality of life in older adults with mild cognitive impairment: Preliminary findings. Alzheimers Dement 12, P310.

[38] Wong WP, Hassed C, Chambers R, Coles J (2016) The effects of mindfulness on persons with mild cognitive impairment: Protocol for a mixed-methods longitudinal study. Front Aging Neurosci 8, 156.

[39] Salzberg S (2002) Lovingkindness: The Revolutionary Art of Happiness, Shambhala Publications, Boston.

[40] Nasreddine ZS, Phillips NA, Bédirian V, Charbonneau S, Whitehead V, Collin I, Cummings JL, Chertkow H (2005) The Montreal Cognitive Assessment, MoCA: A brief screening tool for mild cognitive impairment. J Am Geriatr Soc 53, 695-699.

[41] Antony MM, Cox BJ, Enns MW, Bieling PJ, Swinson RP (1998) Psychometric properties of the 42-item and 21-item versions of the Depression Anxiety Stress Scales in clinical groups and a community sample. Psychol Assess 10, 176181.

[42] Walach H, Buchheld N, Buttenmüller V, Kleinknecht N, Schmidt S (2006) Measuring mindfulness-the Freiburg Mindfulness Inventory (FMI). Pers Indiv Differ 40, 15431555.

[43] Hindmarch I, Lehfeld H, De Jongh P, Erzigkeit H (1998) The Bayer Activities of Daily Living Scale (B-ADL). Dement Geriatr Cogn 9, 20-26.

[44] Erzigkeit H, Lehfeld H, Peña-Casanova J, Bieber F, Yekrangi-Hartmann C, Rupp M, Rappard F, Arnold K, Hindmarch I (2001) The Bayer-Activities of Daily Living Scale (B-ADL): Results from a validation study in three European countries. Dement Geriatr Cogn 12, 348-358.

[45] AIHW, The Active Australia Survey: A guide and manual for implementation, analysis and reporting, Australian Institute of Health and Welfare, https://www.aihw.gov.au/ reports/physical-activity/active-australia-survey/contents/ table-of-contents, 16/08/2017.

[46] Brown WJ, Burton NW, Marshall AL, Miller YD (2008) Reliability and validity of a modified self-administered version of the Active Australia physical activity survey in a sample of mid-age women. Aust N Z J Public Health 32, 535-541.

[47] ALSWH, Seventh survey for the women of the $1946-51$ cohort, Australian Longitudinal Study on Women's Health, http://www.alswh.org.au/for-researchers/surveys.

[48] McKellar S, Horsley P, Chambers R, Pullen M, Vandersee P, Clarke C, Callum H, Bauer JD (2008) Development of the 
Diet Habits Questionnaire for use in cardiac rehabilitation. Aust J Prim Health 14, 43-47.

[49] Carlson MC, Parisi JM, Xia J, Xue QL, Rebok GW, Bandeen-Roche K, Fried LP (2012) Lifestyle activities and memory: Variety may be the spice of life. The Women's Health and Aging Study II. J Int Neuropsychol Soc 18, 286-294.

[50] Cohen J (1988) Statistical Power Analysis for the Behavioral Sciences. L. Erlbaum Associates, Hillsdale, N.J.

[51] Ng KST, Chan HY, Wee ST, Goh LG, Nur F, Tan CTY, Cheah IK-M, Chow WL, Feng L, Ho RC-M (2016) Mindful awareness practice (MAP) to improve the cognition of Singaporean elderly with mild cognitive impairment (MCI): A randomized controlled trial (RCT). Alzheimers Dement 12, P1180-P1181.

[52] Hodgins HS, Adair KC (2010) Attentional processes and meditation. Conscious Cogn 19, 872-878.
[53] Hasenkamp W, Barsalou LW (2012) Effects of meditation experience on functional connectivity of distributed brain networks. Front Hum Neurosci 6, 38.

[54] Innes KE, Selfe TK, Khalsa DS, Kandati S (2017) Meditation and music improve memory and cognitive function in adults with subjective cognitive decline: A pilot randomized controlled trial. J Alzheimers Dis 56, 899-916.

[55] Quintana-Hernández DJ, Miró-Barrachina MT, IbáñezFernández IJ, Pino AS-d, Quintana-Montesdeoca MP, Rodríguez-de Vera B, Morales-Casanova D, Pérez-Vieitez MdC, Rodríguez-García J, Bravo-Caraduje N (2016) Mindfulness in the maintenance of cognitive capacities in Alzheimer's disease: A randomized clinical trial. J Alzheimers Dis 50, 217-232. 